\title{
A FLUSH-MOUNT SENSOR PACKAGE FOR A MEMS PIEZOELECTRIC MICROPHONE WITH THROUGH-SILICON-VIAS FOR AIRCRAFT FUSELAGE ARRAYS
}

\author{
T. Reagan ${ }^{1}$, J. R. Underbrink ${ }^{2}, J$. Meloy $^{2}$ and M. Sheplak ${ }^{1 *}$ \\ ${ }^{1}$ Interdisciplinary Microsystems Group, University of Florida, Gainesville, Florida, USA \\ ${ }^{2}$ Boeing, Seattle, Washington, USA
}

\begin{abstract}
This paper presents the design, fabrication and preliminary testing of a flush-mount sensor die and associated package for an aluminum nitride (AIN) based piezoelectric MEMS microphone for aircraft fuselage arrays. The optimal microphone design is determined using composite plate theory in combination with lumped element modeling. Through-silicon-vias are incorporated into the fabrication of the sensor thus eliminating front-side wire bonds and enabling an overall flush surface for the packaged sensor that minimizes flow disturbance.

Frequency response measurements show a comparable sensitivity $(13.1 \mu \mathrm{V} / \mathrm{Pa})$ and significantly higher resonant frequency $(194 \mathrm{kHz})$ than prior piezoelectric aeroacoustic microphones. The developed packaging method for the sensor demonstrates an overall flushness to within $10 \mu \mathrm{m}$, showing substantial improvement from any previously reported efforts. This is the first truly flush-mount piezoelectric MEMS microphone with associated packaging that has been developed.
\end{abstract}

\section{INTRODUCTION}

In their effort to locate, understand and mitigate the impact of noise sources on an aircraft, aeroacoustic researchers are in need of a high performance, low cost microphone to address the increasing noise restrictions on commercial aircraft. Existing commercial sensors, even with their relatively high cost, in some cases constrain the quality and type of measurement that may be achieved.

Previously developed MEMS aeroacoustic microphones [110] have failed to address the need for a sensor that can be packaged and installed with a hydraulically smooth front surface to be used for boundary layer measurements in a fuselage array. Protrusions from the surface must be within the viscous sublayer, or smaller than 5 viscous wall units, in order for the flow to see an essentially flat surface [11]. The surface topography of the sensor and associated package that utilizes wire bonds alone is locally limited to $\sim 130 \mu \mathrm{m}$ [2], and a required protective encapsulant adds to the surface roughness. Additionally, through-silicon via (TSV) transducer implementation has largely been limited to under-bump metallization attachment techniques [12,13] where sensor flushness is not a priority.

This work addresses the limitations of existing MEMS piezoelectric microphones used in aeroacoustic applications by incorporating through-silicon-vias (TSVs) into the fabrication to eliminate the use of front-side wire bonds that affect the flow field and create an overall flush-mount microphone package. Requirements for this microphone include a low noise floor of $<$ $48 \mathrm{~dB}$ SPL, a flat frequency response function over the audio range from $20 \mathrm{~Hz}-20 \mathrm{kHz}$, a linear response up to $172 \mathrm{~dB}$, and the packaged sensor flushness to be within $12.5 \mu \mathrm{m}$.

\section{DESIGN}

\section{Structure}

The microphone structure includes a circular diaphragm composed of the device layer of a silicon-on-insulator (SOI) wafer, with an annular ring composed of a AlN and molybdenum (Mo) film stack as shown in Figure 1. It also contains a passivation layer to repel moisture, and is front-side vented. The diaphragm displaces under an incident acoustic pressure into the cavity on the backside of the wafer and the resultant strain in the piezoelectric material produces a proportional output voltage across the electrodes; this is the microphone output.

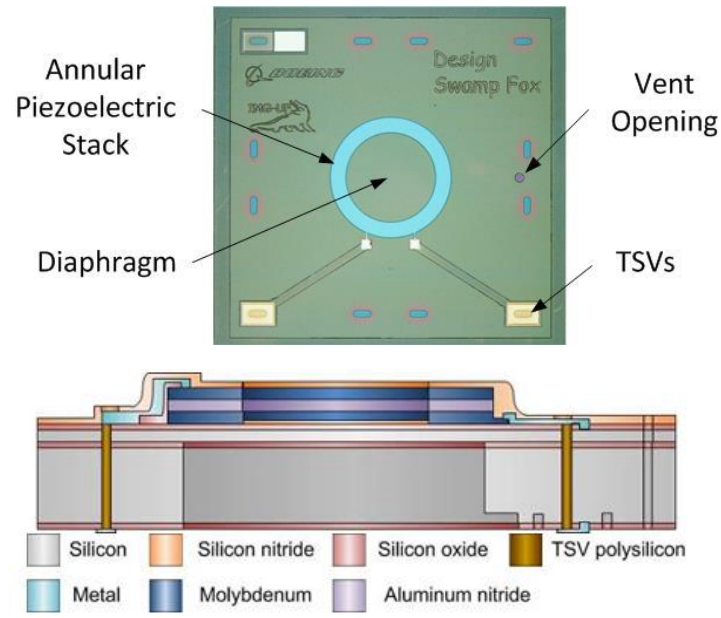

Figure 1: Photograph of an AlN piezoelectric microphone die with $T S V s$, die size is $2 \mathrm{~mm} \times 2 \mathrm{~mm}$ (top) and cross-sectional view of the microphone structure (bottom).

\section{Modeling and Optimization}

The goal of the microphone design is to maximize its operation range in terms of pressure and frequency while meeting the demands of the particular application. The main competing requirements are to achieve a high maximum linear pressure, $P_{\max }$, of $172 \mathrm{~dB}$ while achieving the lowest possible minimum detectable pressure, $M D P$. This is accomplished through the use of the lumped element modeling technique [14] in combination with a piezocomposite diaphragm model [1]. Both are then integrated into a single-objective optimization algorithm that places constraints on the bandwidth and $P_{\max }$, with the objective to minimize $M D P$, where the design variables include the piezoelectric film stack geometry and diaphragm dimensions. Fabrication constraints are also included. The optimization yields a $6.8 \mu \mathrm{m}$ thick diaphragm with an outer diameter of $744 \mu \mathrm{m}$, a $93 \mu \mathrm{m}$ wide piezoelectric film stack and an AlN layer thickness of $1 \mu \mathrm{m}$.

\section{FABRICATION}

Fabrication of the microphones is broken down into three phases, and is outlined in Figure 2. Phase I includes the fabrication of the initial SOI substrate with TSVs and the deposition of the piezoelectric film stack, both of which are performed at external foundries, Icemos Technology Ltd and OEM Group respectively. Phase II is the front-side processing done at the Nanoscale Research Facility (NRF) at the University of Florida. Phase III is the back-side processing steps performed at both NRF and the Lurie Nanofabrication Facility (LNF) at the University of 


\section{Michigan.}

Phase I begins with the formation of TSVs on a SOI substrate using an etch, isolation and deposition process [12]. The formation of the TSVs includes the growth of a $1 \mu \mathrm{m}$ layer of thermal silicon dioxide passivation layer between the substrate and the doped poly-silicon that forms the TSV. When this is done, oxide is also formed on the front and back surfaces of the wafer. The front layer is retained in diaphragm composition (Figure 2a). Thereafter, the $\mathrm{Mo} / \mathrm{AlN} / \mathrm{Mo}$ piezoelectric film stack is deposited (Figure 2b), and the wafers are ready for Phase II processing, which defines the piezoelectric transducer.

Phase II begins by defining the piezoelectric annular ring. A thin layer of silicon dioxide is deposited, and then a small feature is defined to provide electrical isolation between the two electrodes, and prevent an electrical short when the front side metal contacts are made. After etching a ground strap to the device layer and performing a metal liftoff to connect the electrodes to the TSVs (Figure 2c), a hydrophobic silicon nitride layer is deposited to protect the sensor from moisture, and the front side vent opening is patterned. Contact windows are etched through the nitride layer to expose the metal pads over the TSVs, and allow for front-side probing (Figure 2d).

Phase III first consists of etching a handle layer ground strap, then a back side metallization step to form contact pads to the TSVs. A winding vent channel is defined (Figure 2e) and finally, a deep reactive ion etch process is used to form the cavity and vent opening to the front side (Figure 2f). The resulting sensors are then diced into a final size of $2 \mathrm{~mm} \times 2 \mathrm{~mm}$ using a laser dicing method. (a)

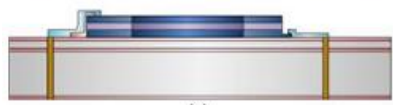

(c)

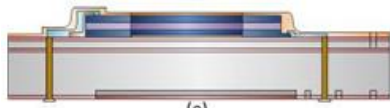

(e)

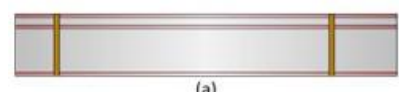

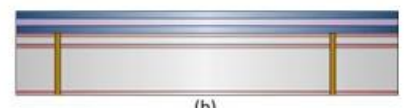

(b)
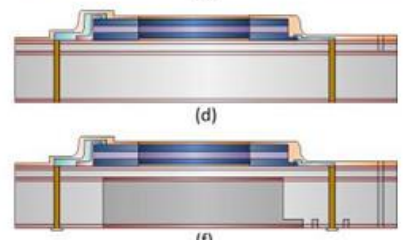

Figure 2: Schematic view of the process steps to create an AlN piezoelectric microphone with TSVS.

\section{PACKAGING}

The final sensor package is comprised of two main components, the endcap assembly and the laboratory test assembly, necessary for the calibration of the sensors.

The "endcap" assembly consists of the sensor die, a circular printed circuit board (PCB) substrate and a circular metal shim cap that sets the sensor flushness as shown in Figure 3. The sensor and shim cap are attached to the PCB using an epoxy film. This film seals the back cavity by encompassing the edges of the die without clogging the vent structure, which prevents unwanted acoustic leakage paths and thereby controls the low frequency response of the sensor with the combination of the electrical properties of the piezoelectric film and the chosen cavity and vent geometries. The electrical connections to the endcap are recessed into the PCB within the perimeter of the die for protection.

The process for attaching the sensor to the PCB is shown in Figure 4. First, the PCB is prepared by planarizing the front and back surfaces. During the PCB manufacture, the gold-plated through holes, or vias, protrude above the surface and must be polished down to create a smooth surface. Next, a pocket is milled on the back side of the board over the vias to create a recess and holes are milled through the PCB to access the metal pads on the back side of the sensor. The epoxy film is then cut to the desired dimensions using laser machining with a cutout for the die in the center and an overall diameter slightly smaller than the shim cap. The shim cap thickness is chosen such that the combined height of the shim cap and epoxy film is $\sim 25$ um thicker than the sensor.

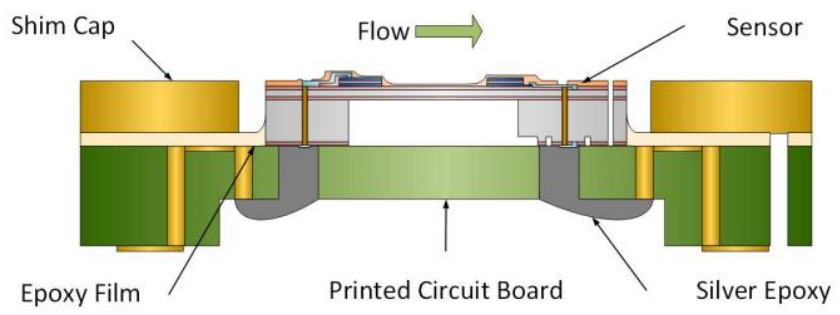

Figure 3: Cross-sectional view of the endcap assembly for a flushmount sensor package.

It is at this point that the sensor and shim cap are placed on a layer of heat release tape to protect the diaphragm. The epoxy film is placed on the PCB. A flip chip bonder is then used to align and place the sensor/shim cap combination on the PCB. Pressure is applied during this process to seat the sensor on the PCB surface. During this process, the shim cap pushes down on the epoxy film, encasing the sensor around its perimeter. The chuck of the flip chip bonder ensures that the shim cap and sensor top surface remain at the same height and establish a hydraulically smooth front surface. Once heat cured, silver epoxy is used to fill the drilled holes on the PCB and connect the back side contacts of the sensor to the vias on the endcap.
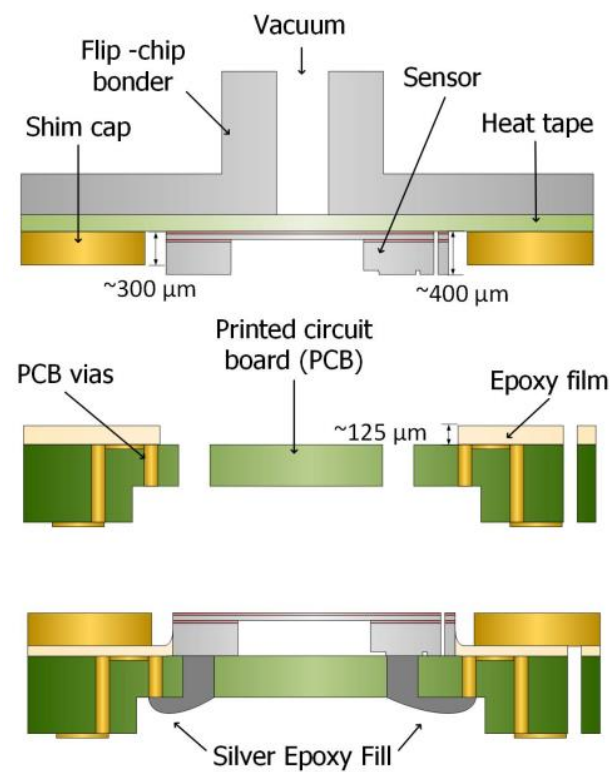

Figure 4: Assembly steps for a flush-mount sensor package.

In order to facilitate testing, the endcap assembly is then mounted onto a larger fixture (the laboratory test assembly) to provide structural support and electrical connections (Figure 5). The endcap assembly is secured to the top of a brass tube which houses an additional PCB with a buffer amplifier and filter capacitors. The amplifier board is interfaced to the endcap assembly with solder connections. Wire connections from the amplifier board for amplifier power and output emerge from the back of the brass tube and are stress relieved using heat shrink 
tubing. Finally, an outer nylon sleeve is used for electrical isolation and to ensure mounting flushness in the test fixture.

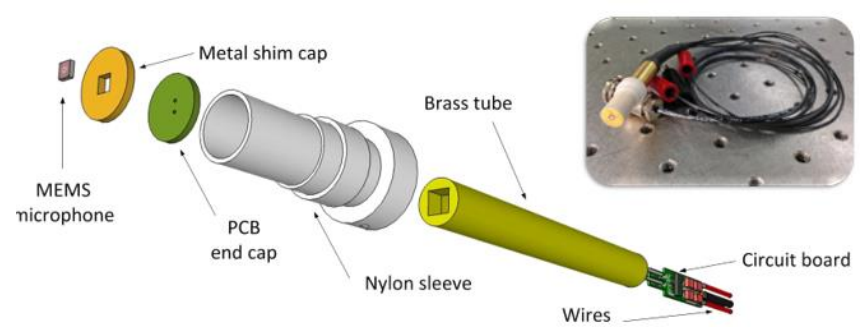

Figure 5: The fully assembled laboratory test package showing the endcap assembly, brass tube and interface circuitry (left); and a photograph of the assembled device (right).

\section{EXPERIMENTAL SETUP AND RESULTS}

This section describes the preliminary experimental characterization of the microphone as well as the package. Measurements of the surface roughness of the endcap assembly, resonant frequency and sensitivity are presented.

\section{Surface Roughness}

Once packaged, the surface roughness of selected device is quantified using a Bruker Contour GT-1 scanning white light interferometer (SWLI). Multiple measurements are stitched together to form a complete scan of the entire endcap assembly and are shown in Figure 6. An overall flushness of $\sim 10 \mu \mathrm{m}$ is achieved with a sensor to shim cap step height of $\sim 8 \mu \mathrm{m}$.

\section{Resonant Frequency}

The voltage chirp excitation response of the microphone is measured using a Polytec scanning laser vibrometer system. The pre-packaged measured resonant frequency of $194 \mathrm{kHz}$ compares favorably with the predicted value of $181 \mathrm{kHz}$ when accounting for fabrication uncertainties. The resonant frequency also provides a qualitative measure of the bandwidth of the device in lieu of an explicit high frequency acoustic characterization method that will be conducted at a later date. The post-packaged measured resonant frequency of $237 \mathrm{kHz}$ indicates a significant amount of thermal stress is imparted to the sensor during the packaging process, which indicates a reduction in achievable sensor sensitivity. An epoxy film specifically designed for substrates with mismatched coefficients of thermal expansion has since been found, and will be tried at a later date to assess its mitigation on the impact of thermal stresses incurred during the packaging process.

\section{Sensitivity}

The frequency response of the device under test (DUT) is determined through comparison with a measurement-grade reference microphone in an acoustic plane wave tube (PWT). A PWT is a rigid duct that allows for the propagation of acoustic plane waves, such that microphones mounted at the same lengthwise location along the duct will see the same pressure, provided that the drive frequency is below the cutoff frequency, $f_{c}$. This frequency is dependent upon the isentropic speed of sound of the medium and the cross section of the duct.

The experimental setup can be seen in Figure 7, where the DUT and the reference, a Bruel and Kjaer 4138 1/8-in pressure field microphone, are mounted at the end of the PWT. An Agilent 33220A Function Generator in combination with a National Instruments PXI Data Acquisition system is used to generate the test signal and acquire the data. The pseudo-random noise test signal is sent through a Crown XLS 1500 amplifier before reaching a BMS 4590 compression driver. Measurements are conducted over a $6.4 \mathrm{kHz}$ bandwidth using a $1 \mathrm{~Hz}$ bin width and a center frequency of $3.5 \mathrm{kHz}$. The maximum frequency of $6.7 \mathrm{kHz}$ is the cutoff frequency for the PWT, and the lower limit of $300 \mathrm{~Hz}$ is dictated by the poor response of the speaker below that frequency.

The frequency response measurement for the selected microphone is shown in Figure 8 in terms of magnitude and relative phase. The magnitude is flat to within $1.5 \mathrm{~dB}$ and the sensitivity taken at $1 \mathrm{kHz}$ is $13.1 \mu \mathrm{V} / \mathrm{Pa}(-97.6 \mathrm{~dB}$ ref $1 \mathrm{~V} / \mathrm{Pa})$.
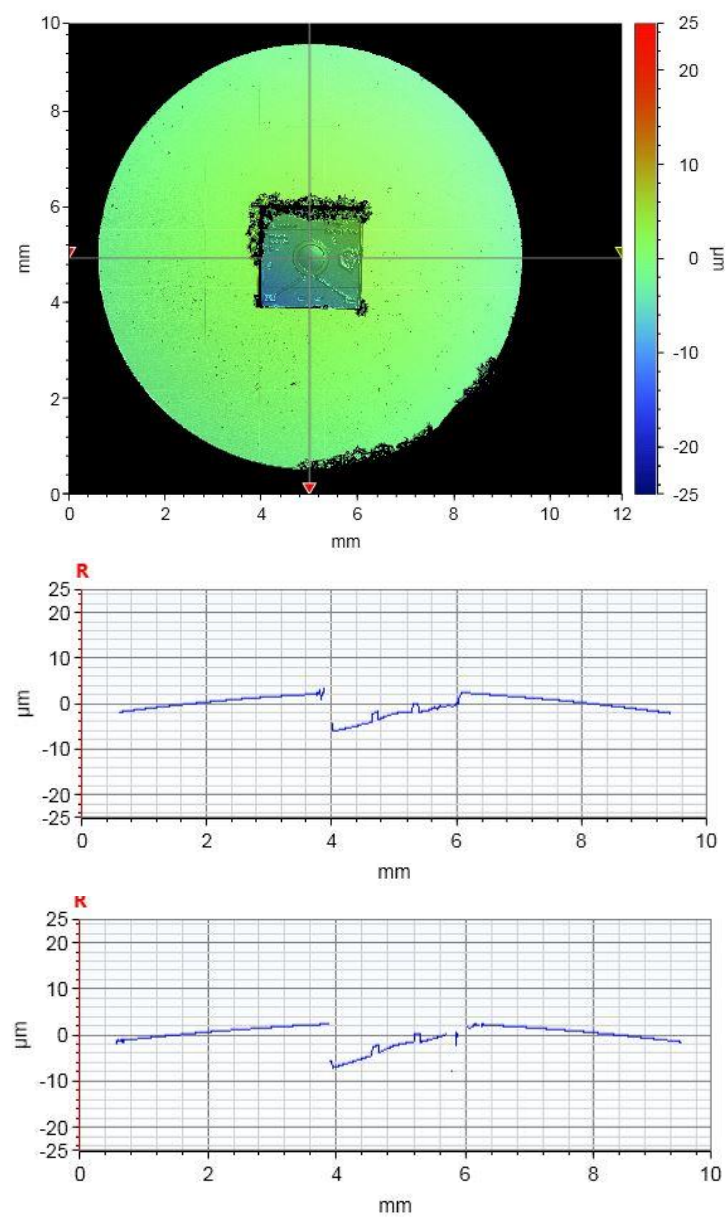

Figure 6: SWLI measurement of assembled endcap structure; Top view (top), X-profile (middle) and Y-profile (bottom).

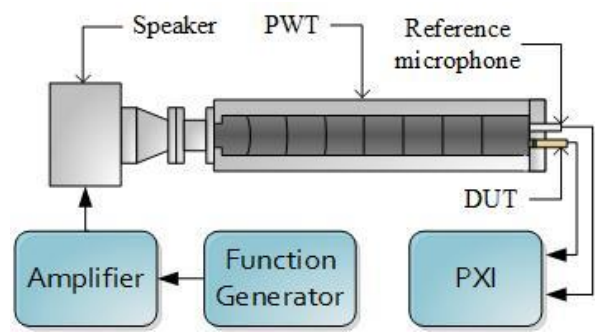

Figure 7: Frequency response measurement experimental setup.

\section{Thermal Cycling Analysis}

The sensor endcap assembly integrity with regards to thermal cycling is evaluated in order to assess the reliability of the package. The mismatch in coefficients of thermal expansion (CTE) between the PCB and sensor generate high stresses and strains at the attachment point when rigidly connected, such as with a solder 
joint [15].

Thermal stability is evaluated by cycling the microphone package from room temperature to $125^{\circ} \mathrm{C}$, down to $-55^{\circ} \mathrm{C}$ and back to room temperature multiple times in an Espec ESX-3CA environmental chamber. The frequency response is then measured and compared to pre thermal cycling measurements. The applied thermal cycling profile and resulting measurements are shown in Figure 9. The sensitivity of the device taken at $1 \mathrm{kHz}$ after thermal cycling was $12.6 \mu \mathrm{V} / \mathrm{Pa}$, and was within $4 \%$ of the pre-cycling measurement.
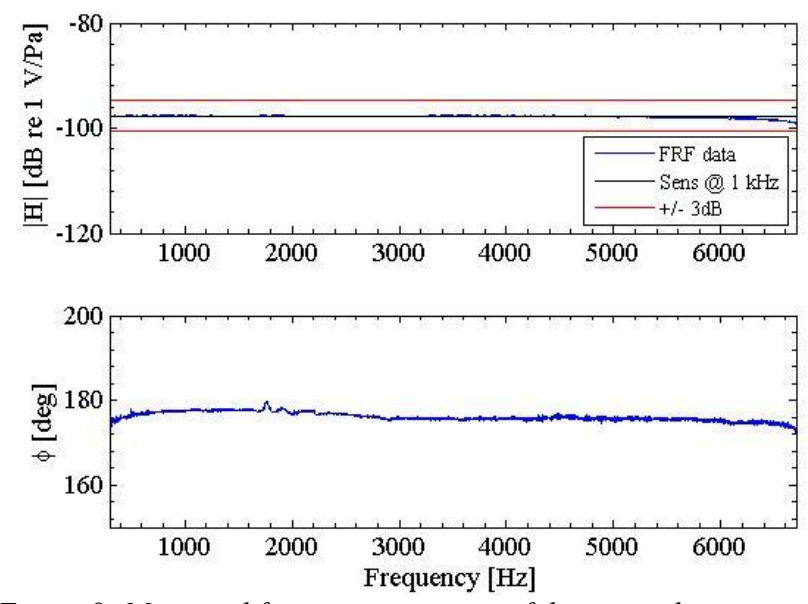

Figure 8: Measured frequency response of the microphone.
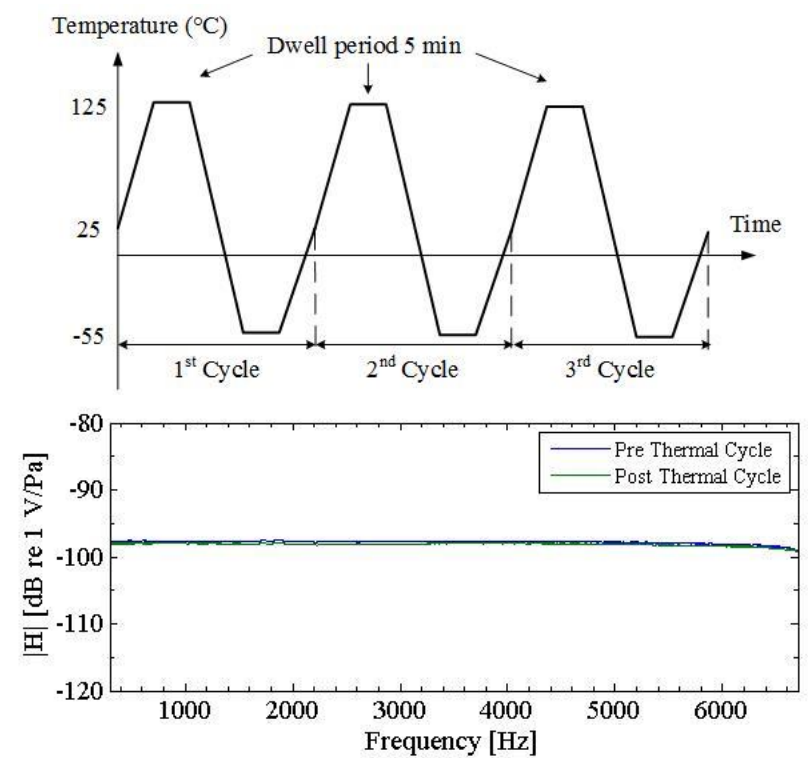

Figure 9: Applied thermal cycling profile (top) and measured sensitivity of the microphone before and after thermal cycling (bottom).

\section{CONCLUSIONS}

A flush-mount piezoelectric microphone and associated package for aircraft fuselage array measurements has been developed. TSVs were formulated into the fabrication to eliminate the use of front side wire bonds. The packaged sensor demonstrates an overall flushness to within $10 \mu \mathrm{m}$, showing an order of magnitude improvement from recently reported efforts in similar endeavors [2]. Preliminary characterization shows a comparable sensitivity $(13.1 \mu \mathrm{V} / \mathrm{Pa})$ and significantly higher resonant frequency $(194 \mathrm{kHz})$ than the most recent piezoelectric aeroacoustic microphone [1]. This piezoelectric microphone and associated package is the first to exhibit a truly flush front surface.

\section{ACKNOWLEDGEMENTS}

This material is based upon work supported by the National Science Foundation Graduate Research Fellowship under Grant No. DGE-0802270. Additional support has been provided by Boeing and the NSF I/UCRC MIST Center.

\section{REFERENCES}

[1] M. Williams et. al., "An AIN MEMS Piezoelectric Microphone for Aeroacoustic Applications", Journal of Microelectromechanical Systems, 21, 1 (2012).

[2] J. Krause et. al., "A Microphone Array on a Chip for High Spatial Resolution Measurements of Turbulence", Journal of Microelectromechanical Systems, 23, 5 (2014).

[3] Z. Zhou et. al., "Damped Aero-Acoustic Microphone with Improved High-Frequency Characteristics", Journal of Microelectromechanical Systems, 23, 5 (2014).

[4] R. Littrell, "High performance piezoelectric MEMS microphones," Ph.D. dissertation, Univ. Michigan, Ann Arbor, MI, 2010.

[5] D. Martin et. al., "A Surface Micromachined Capacitive Microphone for Aeroacoustic Applications," Technical Digest of the 2008 Solid-State Sensor and Actuator Workshop, Hilton Head Isl., SC, 6/1-6/5, Transducer Research Foundation Cleveland (2008), pp. 1-4.

[6] S. Horowitz et. al., "Development of a Micromachined Piezoelectric Microphone for Aeroacoustics Applications," Journal of the Acoustical Society of America, 122, 6 (2007).

[7] P. Scheeper et. al., "A New Measurement Microphone Based on MEMS Technology," Journal of the Acoustical Society of America, 12, 6 (2003).

[8] C. Huang et. al., "A Silicon Micromachined Microphone for Fluid Mechanics Research," Journal of Micromechanics and Microengineering, 12, 6 (2002).

[9] D. P. Arnold et. al., "A Directional Acoustic Array Using Silicon Micromachined Piezoresistive Microphones," Journal of the Acoustical Society of America, 113, 1 (2003).

[10] M. Sheplak et. al., "A Wafer-Bonded, Silicon-Nitride Membrane Microphone with Dielectrically-Isolated, SingleCrystal Silicon Piezoresistors," Technical Digest of the 1998 Solid-State Sensor and Actuator Workshop, Hilton Head Isl., SC, 6/8-6/11, Transducer Research Foundation Cleveland (1998), pp. 23-26.

[11] N. Hutchins et al, "Hot-Wire Spatial Resolution Issues in Wall-Bounded Turbulence," Journal of Fluid Mechanics, 635 (2009).

[12] E. Chow et. al., "Process Compatible Polysilicon-Based Electrical Through-Wafer Interconnects in Silicon Substrates," Journal of Microelectromechanical Systems, 11, 6 (2002).

[13] D. Lin et al, "Packaging and Modular Assembly of LargeArea and Fine-pitch 2-D Ultrasonic Transducer Arrays," IEEE Transactions on Ultrasonics, Ferroelectrics and Frequency Control, 60 ,7 (2013).

[14] S. Senturia, Microsystem Design, Springer Science+Business Media, Inc., New York, 2001.

[15] J. H. L. Pang et. al., "Thermal Cycling Analysis of Flip-Chip Solder Joint Reliability”, IEEE Transactions on Components and Packaging Technologies, 24, 4 (2001).

\section{CONTACT}

*M. Sheplak, tel: +1-352-392-3983; sheplak@ufl.edu 